\title{
Optimal predictive eco-driving cycles for conventional and electric cars
}

\author{
D. Maamria ${ }^{a}$, K. Gillet ${ }^{a}$, G. Colin ${ }^{a}$, Y. Chamaillard ${ }^{a}$ and C. Nouillant ${ }^{b}$
}

\begin{abstract}
In this paper, the computation of eco-driving cycles for electric and conventional vehicles using receding horizon and optimal control is investigated. The problem is formulated as consecutive-optimization problems aiming at minimizing the vehicle energy consumption under traffic and speed constraints. The solving method is based on Dynamic Programming (DP). The impact of the look-ahead distance on the optimal speed computation is studied to find a trade-off between the optimality and the computation time. Simulation results show that in urban driving conditions, a look-ahead distance of $300 \mathrm{~m}$ to $500 \mathrm{~m}$ leads to a sub-optimality less than $0.6 \%$ in the energy consumption compared to the global solution. For highway driving conditions, a look-ahead distance of $1 \mathrm{~km}$ to $2 \mathrm{~km}$ leads to a sub-optimality less than $0.7 \%$ compared to the global solution.
\end{abstract}

\section{INTRODUCTION}

Nowadays, improving the energy consumption linked to transportation systems is among the major challenges facing the automotive industry. Eco-driving is considered as a major solution to face this challenge. In recent decades, engine technology and car performances have improved rapidly thanking to alternative propulsion technologies and engine downsizing, while drivers have not adapted their behavior (driving style). Eco-driving can be defined as a multi-criteria optimization problem (fuel consumption, trip duration, drivability, etc) of various tasks (navigation, guidance, stabilization) under safety constraints [1]-[3].

The objective of eco-driving is to help the driver to improve his manner of driving in order to reduce the vehicle energy consumption. A human machine interface (HMI) integrated in the dashboard of the vehicle proposes the speed and the gear-box ratio set points to the driver. The computation of these set-points can be formulated as a dynamic optimization problem under some constraints: speed limitations, traffic information, final time and total traveled distance [1], [4][6]. The results reported in the literature show that the energy saving via eco-driving depends on the nature of the trip. In urban driving conditions, the energy saving may reach $20 \%$ while for highway trips, the energy saving is about 5 to $10 \%$ [3], [7]-[10]. Two scenarios depending on the horizon of the available information can be distinguished:

- Off-line scenario: The constraints on the speed limitations and the traffic information are fully known in advance. In this case, an estimation of the upper bound on the energy saving that can be reached through

${ }^{a}$ D. Maamria, K. Gillet, G. Colin and Y. Chamaillard are with Univ. Orléans, PRISME, EA 4229, F45072, Orléans, France. djamaleddine.maamria@gmail.com

${ }^{b} \mathrm{C}$. Nouillant is with PSA Peugeot Citroën, Direction Recherche Innovation \& Technologies Avancées (DRIA), France eco-driving, can be computed through optimal control tools. In addition, heuristic driving rules to improve the vehicle fuel consumption may be extracted from the obtained optimal solution: for example, anticipate traffic flow, drive at a low engine speed and shift up early. This kind of calculation can be, for example, performed using cloud computing. This question was addressed for conventional vehicles in [7], [9], [11], for electric cars in [1], [8], [12], [13] and for hybrid electric cars in [5], [11], [14]-[16].

- On-line scenario: The future driving conditions are partially known for a given time or distance horizon usually called e-horizon. The information can be retrieved from many sources such as ADAS systems, sensors and navigation systems (Global Positioning System GPS unit and satellite navigation). Based on the available information, the optimizer has to find the optimal set points of the vehicle speed and the gear-box ratio in real time. The set points obtained will be sent to the driver or to a Cruise controller [17] (in the case of autonomous cars) and they have to be tracked. This question was mainly studied for trucks (conventional and hybrid) [17]-[20] where the roadway grade is among the biggest contributors to high fuel consumption. The objective was to find the speed and gear-box ratio set-points that minimize a trade-off between the fuel consumption and the trip duration on a predefined speed range. The studies in [21], [22] deal with an ecodriving system using Model Predictive Control (MPC) within a given prediction horizon for passenger cars in an urban road network with complex traffic flows. The study in [23] highlights the potential of real-time vehicle speed optimization as a mechanism for energy saving. A driver feedback system, designed using DP, was demonstrated in a test vehicle that uses the optimal velocity profile for the upcoming road. The prediction horizon was arbitrarily set to $1.5 \mathrm{~km}$.

This paper follows the same approach as the second scenario and pushes it further for passenger (conventional and electric) cars. Depending on the length of the e-horizon, the on-line scenario will lead to a sub-optimality in the energy saving with respect to the global optimum (full knowledge of the future driving conditions). We wish, by using MPC and DP, to select the value of the look-ahead horizon in order to minimize the induced sub-optimality in the energy saving while keeping the algorithm computation time reasonable. For this, various values of the e-horizon are considered and the induced sub-optimality compared to the global optimum 
(where the future driving conditions are known in advance) will be quantified. The choice of the look-ahead distance will be based on the optimality/computation time balance.

The paper is organized as follows. In Section II, the vehicle model is described. The computation of eco-driving cycles is detailed in Section III. Section IV presents the proposed predictive scheme. Numerical and simulation results are discussed in Section $V$ for conventional and electric cars. In light of the results, some conclusions on the choice of the look-ahead distance are drawn.

\section{VEHICLE MODELING}

\section{A. Motion equations}

The vehicle is modeled in a vertical plane. According to Newton's law of motion, the vehicle speed $v$ satisfies the following differential equation

$$
m \cdot \frac{d v(t)}{d t}=F_{t}(t)-F_{r}(t)
$$

where $F_{t}$ is the traction force provided by the prime mover, $F_{r}$ is the sum of resistance forces and $m$ is the total vehicle mass including inertia of rotating parts. The force $F_{r}$ comprises the rolling resistance force, the aerodynamic drag force and a force due to the road grade. Its expression is given by

$$
F_{r}(t)=c_{0}+c_{1} \cdot v(t)+c_{2} \cdot v(t)^{2},
$$

where $c_{i}, i=\{0,1,2\}$ are the coefficients of the road load equation. This model considers only the forces in the longitudinal direction.

\section{B. Prime mover}

In the case of a conventional vehicle, the prime mover is an Internal Combustion Engine (ICE). In this study, the ICE is a Diesel engine. This choice is not restrictive as the approach can be easily extended to gasoline engines. The fuel consumption $\dot{m}_{f}[\mathrm{~g} / \mathrm{s}]$ is computed through a look-up table as a function of the engine torque $T_{e}$ [N.m] and the engine speed $\omega_{e}[\mathrm{rpm}]$ (see Figure 1)

$$
\dot{m}_{f}=\dot{m}_{f}\left(\omega_{e}, T_{e}\right)
$$

In the case of an electric car, the prime mover is an electric machine. It is modeled by a quasi-static map describing the electric power (see Figure 2). This map usually includes the losses in the electric machine and the power electronic devices. The electric power $P_{m}$ is of the form

$$
P_{m}=P_{m}\left(\omega_{e}, T_{e}\right)
$$

\section{Battery model}

The battery is represented by an equivalent circuit model comprising a voltage source $U_{o c v}$ in series with an electric resistance $R_{b}$, both of which vary with $\xi$, the battery state of charge (SOC) [24], [25]. The expression of the battery current $I_{b}$ is given by [24]

$$
I_{b}=\frac{1}{2 R_{b}(\xi)}\left(U_{o c v}(\xi)-\sqrt{U_{o c v}^{2}(\xi)-4 R_{b}(\xi) \cdot P_{m}}\right) .
$$

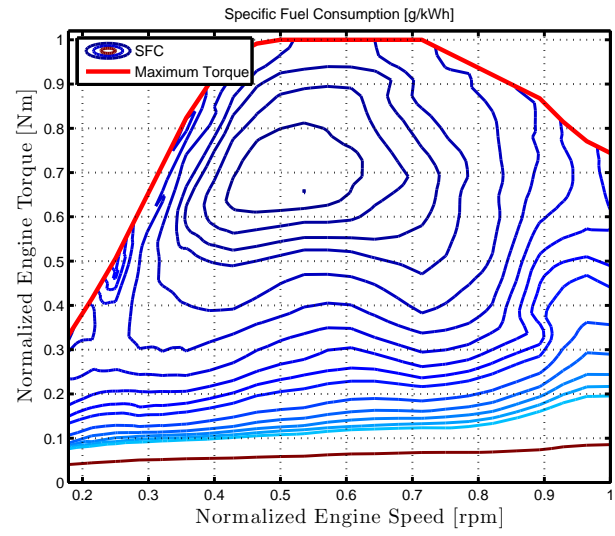

Fig. 1. Specific fuel consumption SFC [g/kWh] of the ICE as a function of the engine torque and engine speed. For confidentiality reasons, the data are normalized.

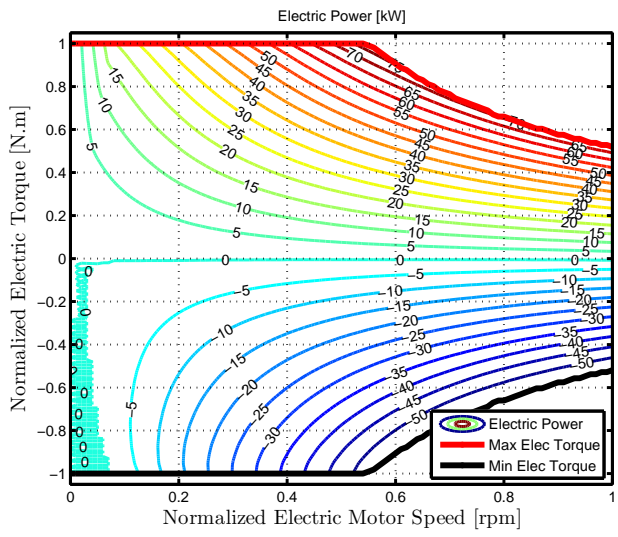

Fig. 2. Electric Machine Power $[\mathrm{kW}]$ as a function of the electric machine torque and electric machine rotational speed.

In the study [13], it was shown that neglecting the dynamics of $\xi$ in the eco-driving cycle computation leads to an acceptable sub-optimality in the energy saving (less than $0.5 \%$ ). The inner (electrochemical) battery power is

$$
P_{e c h}\left(v, T_{e}\right)=I_{b}\left(v, T_{e}\right) \cdot U_{o c v}
$$

The dynamics of $\xi$ is given by

$$
\frac{d \xi(t)}{d t}=-\frac{I_{b}(t)}{Q_{0}}
$$

where $Q_{0}$ is the nominal battery capacity.

\section{Transmission}

The prime mover torque $T_{e}$ is related to the driver's torque demand $T_{w h}$ at the wheel by the relation

$$
T_{w h}(t)=r_{\text {tire }} \cdot F_{t}(t)=\eta_{g b} \cdot R_{g b}(t) \cdot R_{t} \cdot T_{e}(t),
$$

where $R_{g b}$ is the gear-box ratio, $\eta_{g b}$ is the constant gearbox efficiency and $R_{t}$ is the differential ratio. The torque $T_{w h}$ can be positive (traction) or negative (braking). Similarly, the rotational speed $\omega_{e}$ is related to the vehicle speed $v$ by

$$
\omega_{e}(t)=R_{g b}(t) \cdot R_{t} \cdot \frac{v(t)}{r_{\text {tire }}} .
$$


The model parameters are summarized in Table I. The numerical values are omitted for confidentiality reasons.

TABLE I

SYSTEM PARAMETERS

\begin{tabular}{|l|l|c|}
\hline Acronym & Description & Unit \\
\hline$m$ & Vehicle mass & $\mathrm{kg}$ \\
$r_{\text {tire }}$ & Wheel radius & $\mathrm{m}$ \\
$c_{0}$ & Constant coefficient of the road load & $\mathrm{N}$ \\
$c_{1}$ & Linear coefficient of the road load & $\mathrm{N} /(\mathrm{m} / \mathrm{s})$ \\
$c_{2}$ & Quadratic coefficient of the road load & $\mathrm{N} /(\mathrm{m} / \mathrm{s})^{2}$ \\
$\eta_{g b}$ & Gear-box efficiency & - \\
$R_{g b}$ & Gear-box ratio & - \\
$R_{t}$ & Differential ratio & - \\
\hline
\end{tabular}

\section{PROBLEM FORMULATION AND OFFLINE SOLUTION}

For a fixed road, the idea of eco-driving is to find the best vehicle speed profile that minimizes the vehicle energy consumption. The vehicle starts from a point $A$ at a velocity $v_{0}(\geq 0)$ and must reach a destination point $B$ at a predefined time $t_{f}$, with a velocity $v_{1}(\geq 0)$ under some constraints: speed limitations and traffic constraints [6], [8]. This question can be solved using optimal control theory [1], [6].

\section{A. OCP formulation}

The cost function to be minimized over a fixed time window of duration $t_{f}$ is

$$
J=\int_{0}^{t_{f}} L\left(\omega_{e}(t), T_{e}(t)\right) d t
$$

In the case of a conventional vehicle, $L$ is the fuel consumption $\dot{m}_{f}$ given by equation (3) while in the case of an electric car, $L$ is the electrochemical battery power $P_{e c h}$ given by equation (4).

The control variable $u$ is composed of two components: the torque $T_{e}$ and the gear-box ratio $R_{g b}$

$$
u(t)=\left[T_{e}(t), R_{g b}(t)\right]
$$

The considered gear-box has 6 ratios in the case of the conventional vehicle and has only one ratio for the electric vehicle. This optimization problem is carried out under the following dynamical constraints

$$
\begin{array}{lll}
\frac{d v(t)}{d t}=f(v(t), u(t)), & v(0)=v_{0}, \\
\frac{d x(t)}{d t}=v(t), & x(0)=0,
\end{array}
$$

where $x$ is the position of the vehicle and the non-linear function $f$ is calculated by combining $(1,2,5)$.

Due to the limitations of the vehicle speed $v$, of the torque $T_{e}$ and of the gear-box ratio $R_{g b}$, the optimization must be performed under the following mixed state and input constraints

$$
\begin{aligned}
v & \in\left[0, v_{\max }(x)\right], \\
f(v, u) & \in\left[a_{\min }, a_{\max }\right], \\
T_{e} & \in\left[T_{\min }\left(\omega_{e}\right), T_{\max }\left(\omega_{e}\right)\right], \\
\omega_{e} & \in\left[\omega_{\min }, \omega_{\max }\right], \\
x\left(t_{f}\right) & =D, \\
v\left(t_{f}\right) & =v_{1},
\end{aligned}
$$

where $v_{\max }(x)$ is the vehicle speed limitation at position $x$, $D$ is the total traveled distance, $v_{1}$ is the desired final speed, $T_{\min }$ and $T_{\max }$ are the maximum and the minimum torque given by look-up tables as functions of the prime mover rotation speed $\omega_{e}$ (see Figures 1 and 2). In addition, the vehicle acceleration is constrained between its lower and upper bounds ( $\left.a_{\min }, a_{\max }\right)$ in (9).

To summarize, the OCP is

$$
(O C P): \min _{u} \int_{0}^{t_{f}} L(v, u) d t
$$

under the dynamics $(6,7)$, the (mixed) state and input constraints $(8,9,10,11)$, and the final constraints $(12,13)$. Thus, the vehicle speed can be computed by using (6) once the control variable $u$ is optimized and the initial condition of the vehicle speed is known.

\section{B. Speed limitations}

In this study, to specify the speed limitations, a certain (fixed) margin $e_{l}$ on an initial driving cycle speed is considered

$$
v_{\text {max }}(x)= \begin{cases}v(x)+e_{l}, & v(x)>0, \\ 0, & v(x)=0,\end{cases}
$$

where $v(x)$ is the vehicle speed at the position $x$ of the initial driving cycle which is used as a reference of comparison to point out the benefit of eco-driving in terms of fuel consumption reduction. Other methods to compute speed limitations can be considered [7], [14]. For example, the speed limits can be given by the traffic signs (legal speed limits) or provided by a cloud server depending on the vehicle position. It should be pointed out that the speed limits computation method does not impact the solving method.

\section{Solving method}

To solve the (OCP) defined in (14), several methods can be used: Pontryagin Minimum Principle (PMP) or Dynamic Programming (DP). Because of the non-linear nature of the problem under consideration and the mixed state-input constraints (which are activated almost all the time), methods based on PMP are more complicated to implement [26]. For this reason, the method proposed here is based on DP [27], [28]. As suggested in [2], [7], the time-based OCP is transformed into a distance-based OCP in order to reduce the computation time. If the position space is discretized in $N$ fixed steps of length $\Delta x$, the time step $\Delta t(k), k=1: N$ is variable and is calculated from the vehicle speed $v(k)$ and 
the vehicle acceleration $a(k)$ by solving the following second order equation [7]

$$
\Delta x=\frac{1}{2} a(k) \cdot \Delta t(k)^{2}+v(k) \cdot \Delta t(k) .
$$

The final constraint on the vehicle position (12) is fulfilled by construction $(D=N \cdot \Delta x)$. An additional term $\beta \cdot \Delta t(k)$ is added to the cost function as follows:

$$
\bar{J}_{s}(u)=\sum_{k=1}^{k=N}[L(v(k), u(k))+\beta] \Delta t(k) .
$$

The parameter $\beta$ penalizes the final time $t_{f}=\sum_{k=1}^{N} \Delta t(k)$ to obtain almost the same time duration as the initial driving cycle. The studies in [7], [29] show that the relation between $t_{f}$ and $\beta$ is monotone so that $t_{f}$ can be tuned.

\section{Predictive Eco-driving CyCle Computation}

Optimal strategies obtained by DP or any other method are computed using the full knowledge of the future driving constraints (speed limitations and traffic information). In real situations, these future driving conditions are uncertain. They depend on many factors such as local traffic, infrastructure status, non-vehicle actors and weather conditions. To deal with this kind of situation, approaches based on Model Predictive Control (MPC) techniques are considered as an effective solution [30]-[32].

The idea of MPC is the following: the future driving conditions are only known for a look-ahead distance $D_{0} \leq D$ and the speed trajectory will be recalculated every traveled distance $D_{f}$ called optimization frequency where $D_{f}<D_{0}$. The total traveled distance $D$ is divided into $n$ intervals $D_{i}, i=1: n$ where $n$ is the ratio between $D$ and the $D_{f}$. The previous OCP described in (14) will be solved $n$ times

$$
\left(O C P_{i}\right): \min _{u} \int_{t_{i}}^{t_{i+1}} L(v, u) d t
$$

under the dynamics $(6,7)$, the (mixed) state and input constraints $(8,9,10,11)$. The time $t_{i}$ corresponds to the moment when the vehicle reaches the position $\left(i \cdot D_{f}\right)$ while the time $t_{i+1}$ corresponds to the moment when the vehicle reaches the position $\left(i \cdot D_{f}+D_{0}\right)$. Thus, the constraints on the vehicle position are

$$
\begin{gathered}
x\left(t_{i}\right)=i \cdot D_{f}, \\
x\left(t_{i+1}\right)=i \cdot D_{f}+D_{0} .
\end{gathered}
$$

The initial vehicle speed at time $t_{i}$ is given by the final speed reached at the end of the previous segment. The final speed value is free at the end of each segment except for the last segment:

$$
v\left(t_{n}\right)=v_{1},
$$

where $v_{1}$ is the desired final speed at the end of journey.

The optimization scheme is illustrated in Figure 3. The global problem for a traveled distance of $D$ is divided into $n$ sub-problems solved successively. At each traveled distance $D_{f}$, the speed limitations and the traffic information are updated. A new vehicle speed trajectory is calculated for a distance horizon $D_{0}$ by solving the OCP (15) using the method described in Section (III-C). The computed speed trajectory has to be followed by the driver. To avoid the effects due to human interaction, the vehicle is assumed to follow the speed set points precisely (in simulation).

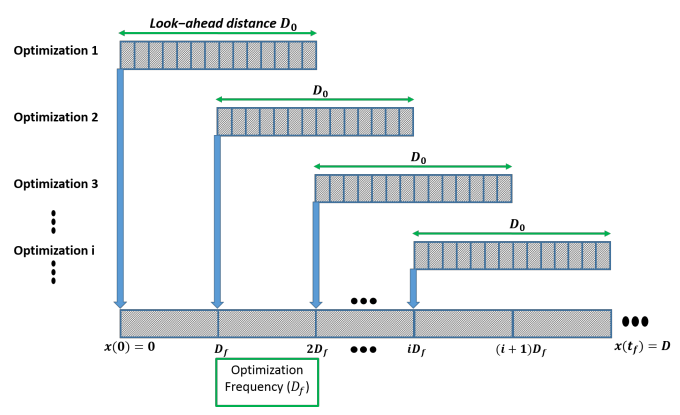

Fig. 3. Predictive algorithm scheme for eco-driving.

Figure 4 shows the inputs, the outputs and the parameters of the algorithm. The inputs are the speed limitations, traffic information and road grade. The outputs of the algorithm are the vehicle speed and the gear-box ratio set points to the low level controllers. The parameter $D_{f}$ controls the launch of the algorithm: its value has to be chosen to find a good trade-off between the optimality of the solution and the computation time. The algorithm can also be launched in the case of sudden events: change in the traffic constraints, appearance of any other barriers (persons crossing the road), difference between real speed and set point. By this methodology, a feedback is introduced.

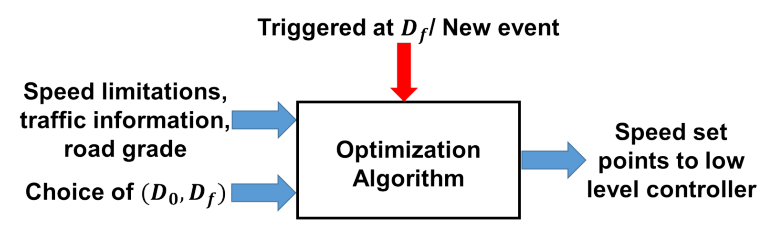

Fig. 4. Algorithm inputs and outputs.

\section{Numerical RESUlts}

Five driving cycles were selected: ECE-15 (Urban Driving Cycle UDC), EUDC (the Extra-urban driving cycle), WLTC (the Worldwide harmonized Light vehicles Test Cycle), the Artemis Urban and Artemis Rural driving cycle [33]. The duration $t_{f}$ without stop phases, the total traveled distance $D$, the final SOC (electric case) and the fuel consumption (conventional case) for each initial driving cycle are given in Table II. The value of $e_{l}$ for the speed limitation was arbitrarily fixed to $2 \mathrm{~km} / \mathrm{h}$.

A standard computer equipped with an Intel Core i5-4590, CPU $3.30 \mathrm{GHz}$ with $8 \mathrm{~GB}$ of RAM was used. The look-ahead distance $D_{0}$ for the six scenarios tested is given in Table III.

Scenario $I$ is considered as a reference of comparison (global optimum) in this study as the look-ahead distance $D_{0}$ equals the total traveled distance: full knowledge of the 
TABLE II

INITIAL DRIVING CYCLES CHARACTERISTICS

\begin{tabular}{|l|c|c|c|c|}
\hline Driving Cycle & $t_{f}[\mathrm{~s}]$ & $D[\mathrm{~km}]$ & $S O C\left(t_{f}\right)[\%]$ & Consumption [g] \\
\hline ECE-15 & 540 & 4 & 83.89 & 202.3 \\
\hline Artemis Urban & 682 & 4.5 & 79.95 & 215.7 \\
\hline Artemis Rural & 1053 & 17.3 & 54.16 & 742 \\
\hline WLTC & 1574 & 22.7 & 37.26 & 973.3 \\
\hline EUDC & 360 & 6.9 & 75.01 & 299.6 \\
\hline
\end{tabular}

TABLE III

$D_{0}[\mathrm{KM}]$ FOR THE VARIOUS SCENARIOS

\begin{tabular}{|c|c|c|c|c|c|c|}
\hline Scenario & $I$ & $I I$ & $I I I$ & $I V$ & $V$ & $V I$ \\
\hline$D_{0}[\mathrm{~km}]$ & $D$ & 4 & 3 & 2 & 1 & 0.5 \\
\hline
\end{tabular}

future driving constraints. The optimization frequency $D_{f}$ was arbitrarily chosen as follows

$$
D_{f}=\left\{\begin{array}{l}
D, \text { for Scenario } I, \\
\frac{D_{0}}{2}, \text { otherwise }
\end{array}\right.
$$

For the DP parameters, a distance step of $\Delta x=10 \mathrm{~m}$ was used in the case of the ECE and the A. Urban and $\Delta x=20 \mathrm{~m}$ for the other driving cycles. This value of $\Delta x$ was chosen so as to get a time step approximately around 1s [29]. The value of $\beta$ is tuned iteratively such that all the eco-driving cycles and the initial driving cycles have almost the same duration (with an error $\leq 0.5 \%$ on the final time $t_{f}$ ). This constraint on the trip duration allows us to make a fair comparison between the initial and the computed eco-driving cycles.

The objective in this study is to find the most suitable choice of $D_{0}$ for which the sub-optimality induced by the receding horizon strategy with respect to the global solution is reduced while keeping the computation time reasonable. The final aim is an embedded algorithm to compute the optimal vehicle speed set-points. Two cases are studied: electric and conventional.

\section{A. Case of an electric car}

The cost function $L$ is the electrochemical battery power $P_{\text {ech }}$. The control variable is the electric machine torque $T_{e}$. The following mesh parameters were used: $0.02 \mathrm{~m} / \mathrm{s}$ for the vehicle speed and $2 \mathrm{~N}$.m for the electric machine torque. The initial value of the SOC was $90 \%$.

For scenario $I$, the results in terms of energy consumption, energy saving through eco-driving with respect to the initial driving cycle and computation time $\alpha$ are given in Table IV.

TABLE IV

RESUlTS OF SCENARIO I (GLOBAL OPTIMUM): ELECTRIC CASE

\begin{tabular}{|l|c|c|c|}
\hline & $S O C\left(t_{f}\right)[\%]$ & Energy reduction $[\%]$ & $\alpha[\mathrm{s}]$ \\
\hline ECE-15 & 85.09 & 19.6 & 3.5 \\
\hline A. Urban & 84.63 & 46 & 4.6 \\
\hline A. Rural & 59.74 & 15.6 & 16.2 \\
\hline WLTC & 50.29 & 24.7 & 24.3 \\
\hline EUDC & 76.86 & 12.3 & 6.8 \\
\hline
\end{tabular}

Figure 5 summarizes the sub-optimality in [\%] of the tested scenarios with respect to scenario $I$. The extra-energy consumption with respect to scenario $I$ increases when the look ahead distance $D_{0}$ decreases, and its maximum value is less than $1.4 \%$. It should be pointed out that the energy saving of scenario $I$ with respect to the initial driving cycle is at least $12.3 \%$ (see Table IV): in the worst case, the energy saving with respect to the initial driving cycle is $10.9 \%$.

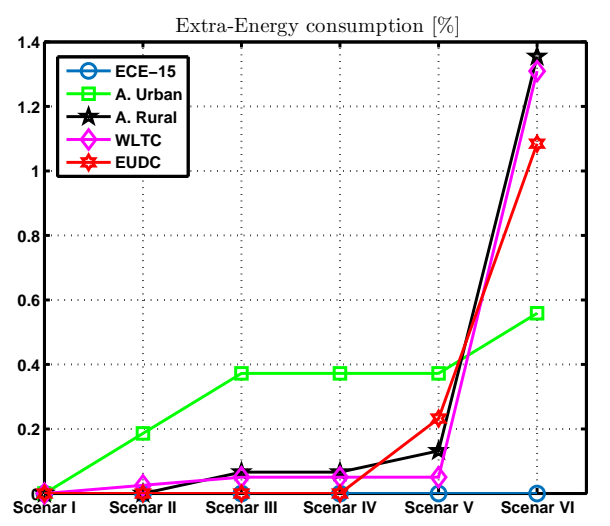

Fig. 5. Sub-optimality in the energy consumption [\%] versus scenarios of Table III: Electric case.

Two cases are distinguished:

- Driving cycles with low mean speed value (ECE-15 and A. Urban): the maximum sub-optimality is less than $0.6 \%$ for scenario $V I$. When $D_{0}=0.3 \mathrm{~km}$ and $D_{f}=$ $0.15 \mathrm{~km}$ (case not presented in Table III), the suboptimality reaches $0.9 \%$ for the ECE- 15 .

- Driving cycles with high mean speed value (A. Rural, WLTC, EUDC): scenario $V$ where the sub-optimality is less than $0.3 \%$ can be considered as a good choice.

The vehicle speed trajectories for the Artemis Rural cycle are given in Figure 6. When $D_{0} \geq 1 \mathrm{~km}$, the eco-driving speed trajectories are similar. When $D_{0}<1 \mathrm{~km}$, a problem of vehicle speed drops appears because the vehicle speed is free at the end of each segment. The value of $D_{f}$ has to be decreased in order to minimize this phenomenon.

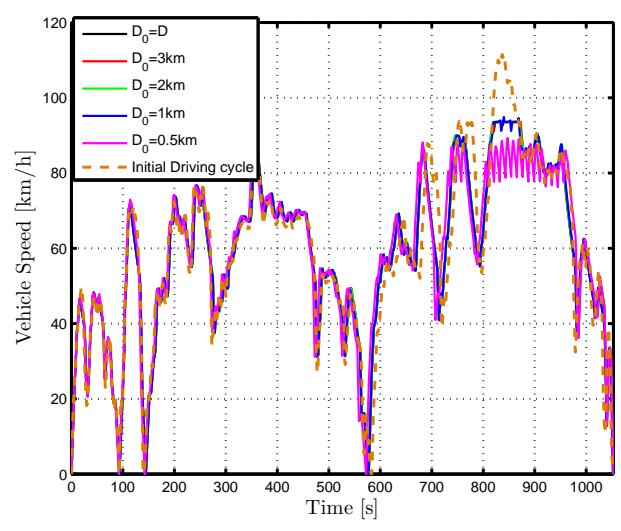

Fig. 6. Vehicle Speed $[\mathrm{km} / \mathrm{h}]$ for the Artemis Rural cycle: Electric case. 
The mean computation time for each optimization and the number of optimizations $n$ for the various scenarios are given in Table V. These mean time values represent the time needed to get the new speed trajectory at each iteration. The number of optimizations represents the load of the CPU and the electric energy consumption associated to this use during the trip. As can be seen from this table, the mean computation time is low and approximately constant for each scenario (in the same column): its value for scenarios $V$ (EUDC, A. Rural and WLTC cases) and VI (ECE-15 and A. Urban cases) is $0.5 \mathrm{~s}$ in the worst case (the time needed to travel $7.5 \mathrm{~m}$ if vehicle speed is $50 \mathrm{~km} / \mathrm{h}$ and $15 \mathrm{~m}$ if vehicle speed is $100 \mathrm{~km} / \mathrm{h}$ ). This time is always lower than the time needed to travel a distance $\Delta x$ (the distance step in the DP).

TABLE V

MEAN COMPUTATION TIME [S] FOR EACH ITERATION AND THE NUMBER OF OPTIMIZATIONS $n$ (BETWEEN BRACKETS) FOR THE VARIOUS SCENARIOS: ELECTRIC CASE

\begin{tabular}{|l|c|c|c|c|c|}
\hline & $I I$ & $I I I$ & $I V$ & $V$ & $V I$ \\
\hline ECE-15 & $1.7(3)$ & $1.8(3)$ & $1.2(5)$ & $0.7(9)$ & $0.4(17)$ \\
\hline A. Urban & $2.1(3)$ & $2.3(3)$ & $1.4(5)$ & $0.8(9)$ & $0.4(18)$ \\
\hline A. Rural & $2.6(9)$ & $1.9(12)$ & $1.1(18)$ & $0.5(35)$ & $0.3(67)$ \\
\hline WLTC & $2.6(12)$ & $1.8(16)$ & $1.2(23)$ & $0.5(46)$ & $0.3(88)$ \\
\hline EUDC & $2.4(4)$ & $1.8(5)$ & $1.1(7)$ & $0.5(14)$ & $0.3(27)$ \\
\hline
\end{tabular}

\section{B. Case of a Conventional vehicle}

The cost function $L$ is the fuel consumption $\dot{m}_{f}$. The control variables are the engine torque $T_{e}$ and the gear-box ratio $R_{g b}$. The following mesh parameters are chosen based on the study [29]: $\Delta v=0.1 \mathrm{~m} / \mathrm{s}$ for the vehicle speed and $\Delta T_{e}=2 \mathrm{~N} . \mathrm{m}$ for the engine torque.

For scenario $I$, the results in terms of fuel consumption, fuel saving through eco-driving with respect to the initial driving cycle and computation time $\alpha$ are given in Table VI.

TABLE VI

RESULTS OF SCENARIO I (GLOBAL OPTIMUM): CONVENTIONAL CASE

\begin{tabular}{|l|c|c|c|}
\hline & Consumption [g] & Fuel saving [\%] & $\alpha[\mathrm{s}]$ \\
\hline ECE-15 & 125.6 & 37.9 & 5.8 \\
\hline A. Urban & 140 & 35.1 & 7.6 \\
\hline A. Rural & 592.3 & 19.9 & 27.1 \\
\hline WLTC & 755.9 & 22.3 & 41 \\
\hline EUDC & 246.2 & 17.8 & 11.5 \\
\hline
\end{tabular}

The induced sub-optimality in [\%] with respect to scenario $I$ is given in Figure 7. The extra-fuel consumption increases when $D_{0}$ decreases, and its maximum value is less than $3.2 \%$. In the case of the ECE-15 and the Artemis Urban, the sub-optimality is less than $0.2 \%$ for all the considered scenarios. In the case of the Artemis Rural, the WLTC and the EUDC, scenario $V$, where the sub-optimality is less than $0.7 \%$, is considered as a reasonable choice. It should be pointed out that the fuel consumption reduction of scenario $I$ with respect to the initial driving cycle is at least $17 \%$ (see

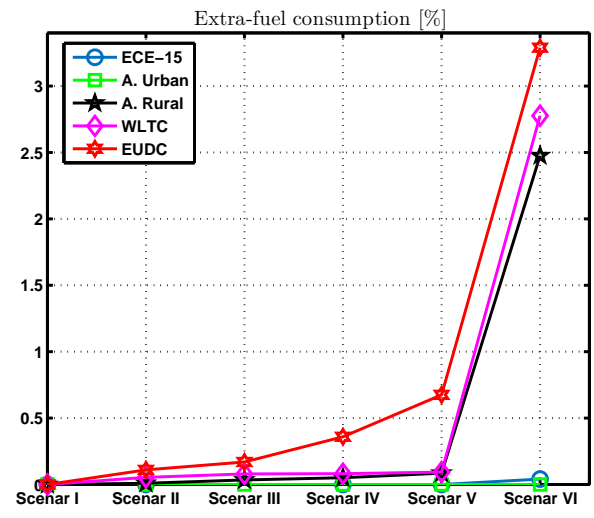

Fig. 7. Extra-fuel consumption [\%] versus scenarios of Table III: Conventional case.

Table VI): in the worst case, the fuel saving with respect to the initial driving cycle is reduced to $14 \%$.

The vehicle speed trajectories for the EUDC case are given in Figure 8. The speed trajectories are similar for $D_{0} \geq 2 \mathrm{~km}$. When $D_{0}$ is lower than $2 \mathrm{~km}$, a problem of

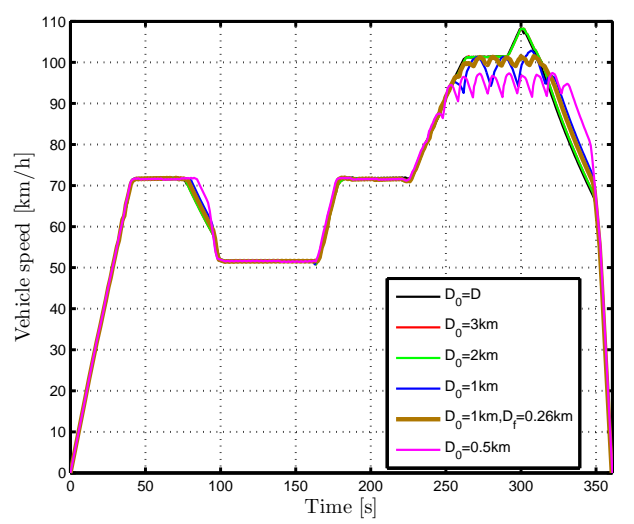

Fig. 8. Vehicle Speed $[\mathrm{km} / \mathrm{h}]$ for the EUDC cycle: Conventional case.

vehicle speed drops appears as in the electric case. To reduce this phenomenon, the value of $D_{f}$ must be decreased when $D_{0}<=1 \mathrm{~km}$ as illustrated in Figure 8 where $D_{0}=$ $1 \mathrm{~km}$ and $D_{f}=0.26 \mathrm{~km}$. The sub-optimality is also reduced: for $D_{f}=0.5 \mathrm{~km}$, the sub-optimality is $0.7 \%$ and for $D_{f}=$ $0.26 \mathrm{~km}$, the sub-optimality is $0.5 \%$. On the other hand, the number of iterations $n$ increases from 14 for $D_{f}=0.5 \mathrm{~km}$ to 27 for $D_{f}=0.26 \mathrm{~km}$.

The number of optimizations $n$ and the mean computation time for each optimization for the various scenarios are given in Table VII. For scenarios $V$ (case of the EUDC, the A. Rural and the WLTC) and VI (case of the ECE-15 and the A. Urban), the computation time is about $1 \mathrm{~s}$ in the worst case: the time needed to travel $28 \mathrm{~m}$ if the vehicle speed is $100 \mathrm{~km} / \mathrm{h}$. This time is always lower than the time needed to travel a distance of $2 \Delta x$ (the distance step in the DP). This result is promising to embed the proposed algorithm. 
TABLE VII

MEAN COMPUTATION TIME [S] FOR EACH ITERATION AND THE NUMBER OF OPTIMIZATIONS $n$ (BETWEEN BRACKETS) FOR THE VARIOUS SCENARIOS: CONVENTIONAL CASE

\begin{tabular}{|l|c|c|c|c|c|}
\hline & $I I$ & $I I I$ & $I V$ & $V$ & $V I$ \\
\hline ECE-15 & $2.9(3)$ & $3(3)$ & $2(5)$ & $1.2(9)$ & $0.7(17)$ \\
\hline A. Urban & $3.6(3)$ & $4(3)$ & $2.5(5)$ & $1.4(9)$ & $0.7(18)$ \\
\hline A. Rural & $4.6(9)$ & $3.4(12)$ & $2.1(18)$ & $1(35)$ & $0.5(67)$ \\
\hline WLTC & $4.6(12)$ & $3.3(16)$ & $2.1(23)$ & $0.9(46)$ & $0.5(88)$ \\
\hline EUDC & $4.2(4)$ & $3.1(5)$ & $2(7)$ & $0.9(14)$ & $0.4(27)$ \\
\hline
\end{tabular}

\section{CONCLUSION}

The computation of eco-driving cycles for electric and conventional vehicles using receding horizon control was addressed and formulated as an optimal control problem. The objective was to study the impact of the look-ahead distance on the optimality of the solution. The result is that, for the two systems considered, in the case of urban driving cycles (mean speed values less than $35 \mathrm{~km} / \mathrm{h}$ ), a look-ahead distance of $300 \mathrm{~m}$ to $500 \mathrm{~km}$ leads to a sub-optimality less than $0.6 \%$ in the energy consumption compared to the global solution (where the future driving conditions are known). This result is interesting to size sensors that predict future driving conditions. For highway driving cycles, a look-ahead distance of $1 \mathrm{~km}$ to $2 \mathrm{~km}$ can be used as the induced suboptimality in the energy consumption is less than $0.7 \%$. Moreover, the computation times are relatively small (1s in the worst case): lower than the time needed to travel a distance of $30 \mathrm{~m}$. This result is a first promising step for a real-time computation using an Engine Control Unit (ECU).

To embed the algorithm, two parameters have to be tuned: the first parameter is used to reach almost the same final time as the initial driving cycle. The second parameter is the frequency with which the optimization is launched so as to find a trade-off between optimality and computation time. Optimizing the tuning of these two parameters is the subject of current investigations and the first results are promising. It is also planned to extend this study to hybrid electric cars.

\section{REFERENCES}

[1] N. Petit and A. Sciarretta, "Optimal drive of electric vehicles using an inversion-based trajectory generation approach," 18th IFAC World Congress, vol. 18, pp. 14 519-14 526, 2011.

[2] V. Monastyrsky and I. Golownykh, "Rapid computation of optimal control for vehicles," Transportation Research Part B, vol. 27, pp. 219-227, 1993.

[3] S.-Y. Kim, D.-J. Shin, and H.-J. e. a. Yoon, "Development of ecodriving guide system," In SAE asia pacific automotive engineering conference, pp. 1636-1641, 2011.

[4] E. Ozatay, U. Ozguner, J. Michelini, and D. Filev, "Analytical solution to the minimum energy consumption based velocity profile optimization problem with variable road grade?" 19th World Congress, pp. 7541-754, 2014.

[5] E. Hellstrom, J. Aslund, and L. Nielsen, "Design of an efficient algorithm for fuel-optimal look-ahead control," Control Engineering Practice, vol. 18, pp. 1318-1327, 2010.

[6] F. Mensing, E. Bideaux, R. Trigui, J. Ribet, and B. Jeanneret, "Ecodriving: an economic or ecologic driving style?" Transportation Research Part C: Emerging Technologies, vol. 38, pp. 110-121, 2014.

[7] F. Mensing, "Optimal energy utilization in conventional, electric and hybrid vehicles and its application to eco-driving," Ph.D. dissertation, INSA Lyon, 2013.
[8] W. Dib, A. Chasse, P. Moulin, A. Sciarretta, and G. Corde, "Optimal energy management for an electric vehicle in eco-driving applications," Control Engineering Practice, vol. 29, pp. 299-307, 2014.

[9] D. Maamria, K. Gillet, G. Colin, Y. Chamaillard, and C. Nouillant, "Optimal eco-driving for conventional vehicles: simulation and experiment," 20th World Congress of IFAC, 2017.

[10] T. J. Daun, D. G. Braun, C. Frank, S. Haug, and M. Lienkamp, "Evaluation of driving behavior and the efficacy of a predictive eco-driving assistance system for heavy commercial vehicles in a driving simulator experiment," 16th International IEEE Conference on Intelligent Transportation Systems, 2013.

[11] A. Sciarretta, G. D. Nunzio, and L. L. Ojeda, "Optimal ecodriving control: Energy-efficient driving of road vehicles as an optimal control problem," IEEE Control Systems Magazine, vol. 35.5, pp. 71-90, 2015.

[12] M. Miyatake, M. Kuriyama, and Y. Takeda, "Theoretical study on ecodriving technique for an electric vehicle considering traffic signals," in Proc. 9th IEEE Int. Conf. Power Electronics Drive Systems, pp. 733-738, 2011.

[13] D. Maamria, K. Gillet, G. Colin, Y. Chamaillard, and C. Nouillant, "On the use of dynamic programming in eco-driving cycle computation for electric vehicles," IEEE MSC, 2016.

[14] H. Bouvier, G. Colin, and Y. Chamaillard, "Determination and comparison of optimal eco-driving cycles for hybrid electric vehicles," European Control Conference, pp. 142-147, 2015.

[15] T. S. Kim, C. Manzie, and R. Sharma, "Two-stage optimal control of a parallel hybrid vehicle with traffic preview," IFAC Proceedings Volumes, vol. 44, pp. 2115-2120, 2011.

[16] T. van Keulen, B. de Jager, D. Foster, and M. Steinbuch, "Velocity trajectory optimization in hybrid electric trucks," in Proc. American Control Conference, pp. 5074-5079, 2010.

[17] B. Passenberg, P. Kock, and O. Stursberg, "Combined time and fuel optimal driving of trucks based on a hybrid model," 2009 European Control Conference (ECC), 2009.

[18] E. Hellstrom, M. Ivarsson, J. Aslund, and L. Nielsen, "Look-ahead control for heavy trucks to minimize trip time and fuel consumption," IFAC Proceedings Volumes, 2007.

[19] S. H. M. Wang, W. Daamen and B. van Arem, "Driver assistance systems modeling by model predictive control," 15th International IEEE Conference on Intelligent Transportation Systems, 2012.

[20] S. T. K. Neiss and T. Connolly, "Predictive speed control for a motor vehicle," United States Patent US 6,990,401 B2, Daimler-Chrysler AG, 2006.

[21] T. Schwickart, H. Voos, J. R. Hadji-Minaglou, and M. Darouach, "An efficient nonlinear model-predictive eco-cruise control for electric vehicles," IEEE Internat Conference on Industrial Informatics, 2013.

[22] M. Kamal and T. Kawabe, "Eco-driving using real-time optimization," European Control Conference, vol. 21, no. 3, pp. 831-841, 2015.

[23] T. Levermore, M. N. Sahinkaya, Y. Zweiri, and B. Neaves, "Real-time velocity optimization to minimize energy use in passenger vehicles," Energies, 2017.

[24] L. Guzzella and A. Sciarretta, Vehicle propulsion systems. Springer, 2013.

[25] F. Badin, Hybrid Vehicles. Editions TECHNIP, 2013.

[26] P. Malisani, "Dynamic control of energy in buildings using constrained optimal control by interior penalty," Ph.D. dissertation, Ecole Nationale Supérieure des Mines de Paris, 2012.

[27] D. Bertsekas, Dynamic programming and optimal control. Athena Scientific, 2012.

[28] A.-E. Bryson and Y.-C. Ho, Applied optimal control. Ginn and Company: Waltham, MA, 1969.

[29] D. Maamria, K. Gillet, G. Colin, Y. Chamaillard, and C. Nouillant, "Which methodology is more appropriate to solve eco-driving optimal control problem for conventional vehicles?" IEEE MSC, 2016.

[30] M. Back, M. Simons, F. Kirschaum, and V. Krebs, "Predictive control of drivetrains," IFAC 15th triennial world congress, Spain., 2002.

[31] M. Back, S. Terwen, and V. Krebs, "Predictive powertrain control for hybrid electric vehicles," Proc. IFAC Symposium on Advances in Automotive Control, University of Salerno, Italy, 2004.

[32] S. Qin and T. Badgwell, "A survey of industrial model predictive control technology," Control engineering practice, vol. 11, pp. 733764, 2003.

[33] A. Michel, "The ARTEMIS European driving cycles for measuring car pollutant emissions," Science of The Total Environment, vol. 334-335, pp. 73-84, 2004. 